\title{
Use of statistical evaluation of antigen profiles in differential diagnosis between colonic and ovarian adenocarcinomas
}

\author{
S C HENZEN-LOGMANS*, N W SCHIPPER, * L G POELS, $\dagger$ K STOLK, * \\ P KENEMANS $\ddagger$ C J L M MEYER*
}

From the *Department of Pathology, Free University, Amsterdam, and the Departments of $\dagger$ Cytology and Histology and $\ddagger$ Gynaecology, University of Nijmegen, Nijmegen, The Netherlands

SUMMARY A study was carried out to determine whether it was possible to classify colonic and ovarian adenocarcinomas by their antigen profile. Colonic and ovarian adenocarcinomas were immunostained with a panel of antibodies which have a limited specificity for colon (parlam-4, 19.9, anti-secretory component) and ovary (OV-TL3 and OC125) and the most discriminatory antibodies were selected by stepwise linear discriminant analysis. For frozen material OV-TL3 and OC125 were the best classifying antibodies. Although $\mathrm{OC} 125$ had better discriminative power, for paraffin wax embedded material parlam-4 was selected as the best classifying antibody. OC125 had no additional effect on the classification of a tumour. These antibodies were subsequently tested on an independent test set of primary and metastatic adenocarcinomas of colonic and ovarian origin. When ovarian posterior probabilities of $<0.1$ and $>0.9$ were selected as cut off points for a positive identification of colonic or ovarian origin (jackknifed classification method), no adenocarcinoma was incorrectly identified as ovarian carcinoma in frozen material. The same trend was noticed for paraffin wax embedded material.

Statistical analysis of antigen profiles can be helpful in defining the colonic or ovarian origin of an adenocarcinoma when routine microscopy does not yield a definitive result.

Adenocarcinomas form a substantial proportion of all malignant epithelial tumours. Treatment depends on the organ of origin, so it is important to define the primary site in the case of metastatic disease. Although histological examination is usually very helpful, there may be problems in defining the primary site of an adenocarcinoma. This difficulty sometimes occurs in adenocarcinomas of presumed ovarian or colonic origin.

In this study we investigated whether antibodies that reacted with ovary and colon associated antigens could help to define the primary site of an adenocarcinoma. First we tested the antibodies on a "training" set of primary ovarian and colonic adenocarcinomas. The most discriminatory combinations of antibodies were detected by stepwise linear discriminant analysis. The combinations were then used on a different test set of primary and metastatic colonic and ovarian adenocarcinomas to find out whether the colonic or ovarian origin of these tumours could be predicted.

Accepted for publication 9 February 1988

\section{Material and methods}

The study was designed for frozen as well as for paraffin wax embedded material. A "training" set and a test set were made up from both types of material. All material was collected from January 1986 to May 1987.

FROZEN MATERIAL

The "training" set comprised 20 primary colonic adenocarcinomas and 24 primary ovarian adenocarcinomas. ${ }^{\prime}$ Colonic adenocarcinomas were taken from 11 men aged between 46 and 72 years and nine women aged between 38 and 88 years. The age of the 24 women with ovarian adenocarcinomas ranged from 26 to 82 years. The ovarian tumours were classified according to Serov and Scully' into 13 serous tumours and 11 mucinous tumours (indeterminate and adenocarcinomas).

The test set comprised 21 primary colonic adenocarcinomas with eight metastases, and 17 primary ovarian adenocarcinomas with five metastases. 
Colonic adenocarcinomas were taken from 12 men and nine women aged 32 to 78 years. The age of the 17 women with ovarian adenocarcinomas ranged from 35 to 86 years. The primary ovarian tumours were classified as nine serous adenocarcinomas, three mucinous tumours (two adenocarcinomas, one borderline), one mixed serous/mucinous adenocarcinoma, one endometrioid adenocarcinoma, and three indeterminate adenocarcinomas. The metastatic lesions were classified as four serous adenocarcinomas and one indeterminate adenocarcinoma.

Metastatic lesions of colonic adenocarcinomas were found in lymph nodes and liver. No metastases of colonic adenocarcinomas in the ovaries were found. Metastases of ovarian carcinomas were peritoneal and on the serosa of the bowel.

\section{PARAFFIN WAX EMBEDDED MATERIAL}

The training set was the same as that used for the frozen material. The test set comprised 24 primary colonic adenocarcinomas with 11 metastatic lesions, and 23 primary ovarian adenocarcinomas with 17 metastatic lesions. Colonic adenocarcinomas were taken from 14 men and 10 women (aged 32 to 82 years). The age of the 23 women with ovarian adenocarcinomas ranged from 35 to 86 years. The primary ovarian tumours were classified as 11 serous adenocarcinomas, six mucinous tumours (four adenocarcinomas, two borderlines), and six indifferentiated adenocarcinomas. Metastatic lesions were classified as 10 serous adenocarcinomas, three mucinous adenocarcinomas, and four poorly differentiated adenocarcinomas.

Metastatic lesions of colonic adenocarcinomas occurred in lymph nodes and liver. No metastases of colonic adenocarcinomas in the ovaries were found. Metastases of ovarian carcinomas were localised in the peritoneum and in the serosa of the bowel.

When fresh material was received, tissues were snap frozen in liquid nitrogen and stored in liquid nitrogen until use, then fixed in $4 \%$ neutral buffered formalin and subsequently embedded in paraffin wax (melting point $52-54^{\circ} \mathrm{C}$ ).
Details of the antibodies used are given in table 1. Monoclonal antibodies were selected because of their preferential reactivity with adenocarcinomas of either colon (19.9, parlam-4, and antisecretory component) or ovary (OC125 and OV-TL3). Several monoclonal antibodies reactive with mucins $(115 \mathrm{D} 8,67 \mathrm{D} 11$, and B 72.3) and with an as yet undefined antigen present on adenocarcinomas (125 B5) were also included because of possible differences in immunoreactivity of these antibodies with colonic or ovarian adenocarcinomas.

For immunohistochemical staining both frozen and paraffin wax sections were stained by the indirect immunoperoxidase method. ${ }^{16}$ Monoclonal antibodies 125 B5 and OV-TL3 were used only on frozen sections as the reactive epitopes not resistant to formalin fixation. All other antibodies were used on both frozen and paraffin wax sections. OV-TL3 was preferentially used in the indirect immunofluorescence method as considerable background staining hampered interpretation. After incubation and washing a goat antimouse antibody labelled with fluorescein isothiocyanate was used as a second layer antibody. The sections were then washed in phosphate buffered saline (PBS), mounted in Fluoromount, and examined with a Leitz fluorescence microscope at an excitation wave length of $490 \mathrm{~nm}$ and an emission wave length of $525 \mathrm{~nm}$. For both immunohistochemical methods, known positive controls were included in each staining series. PBS and non-immune ascitic fluid were used as negative controls.

\section{SCORING OF THE STAINING RESULTS}

In each staining series, and for each antibody, positive epithelial cells were semiquantitatively estimated as previously described. ${ }^{17}$ Staining of secretion products in the lumina of glandular structures was not scored. The percentage of positive staining tumour cells was recorded in decimal notation: $0-10-20$ etc, up to $100 \%$. The pattern of staining was also noted-apical, membranous, basal, diffuse.

The staining results obtained with the respective antibodies were analysed separately by univariate and multivariate analysis for frozen material and paraffin

Table 1 Antibodies used in this study

\begin{tabular}{|c|c|c|c|}
\hline Antibody & Antigen & Preference & Reference \\
\hline $\begin{array}{l}\text { OC125 } \\
\text { OV-TL3 } \\
19.9\end{array}$ & $\begin{array}{l}\text { CA 125, mucin-like glycoprotein, molecular weight }>200 \mathrm{kd} \\
\text { OA3, molecular weight } 80-95 \mathrm{kd} \\
\text { CA } 19.9 \text {, monosialoganglioside (cell associated), mucin (in circulation), } \\
\text { mw? }\end{array}$ & $\begin{array}{l}\text { Ovary } \\
\text { Ovary } \\
\text { Colon }\end{array}$ & $\begin{array}{l}2-4 \\
5 \\
6\end{array}$ \\
\hline $\begin{array}{l}\text { Parlam-4 } \\
\text { Anti-secretory component } \\
115 \text { D8 } \\
67 \text { D11 } \\
125 \text { B5 } \\
\text { B } 72.3\end{array}$ & $\begin{array}{l}\text { Carcinoembryonic antigen, glycoprotein, molecular weight } 180 \mathrm{kd} \\
\text { Secretory component, glycoprotein, molecular weight } 60-70 \mathrm{kd} \\
\text { MAM-6, glycoprotein, molecular weight }>400 \mathrm{kd} \\
\text { MAM-3, glycoprotein, mw? } \\
\text { Unknown } \\
\text { TAG-72, glycoprotein, molecular weight } 220-400 \mathrm{kd}\end{array}$ & $\begin{array}{l}\text { Colon } \\
\text { Colon } \\
\text { Pan-carcinoma } \\
\text { Pan-carcinoma } \\
\text { Unknown } \\
\text { Pan-carcinoma }\end{array}$ & $\begin{array}{l}7 \\
8 \\
910 \\
11 \\
13 \\
14 \\
14\end{array}$ \\
\hline
\end{tabular}

mw? = molecular weight not known. 
wax embedded material. Univariate analysis of the training set was performed for each antibody separately using Wilcoxon's non-parametric test and the $\chi^{2}$ test (programs P3S and P4F of the BMDP statistical software package). A p value of $<0.05$ was regarded as significant.

To determine what combination of antibodies gave the best discrimination between colonic and ovarian adenocarcinomas in the training set a linear stepwise discriminant analysis was carried out (program P7M of the BMDP statistical software package (BMDP Biomedical computer programs, Berkeley, University of California, 1983). Evaluation of the discriminant function, which is a linear combination of two antibodies selected from the training set, was performed by classifying specimens of the test set.

\section{Results}

With most monoclonal antibodies used on paraffin wax or frozen sections a cytoplasmic staining pattern was generally observed in the tumour cells, sometimes with a membranous accentuation. With 115 D8 and OC125 an apical staining pattern was often observed, especially in the better differentiated adenocarcinomas of both colon and ovary. Pronounced heterogeneity of antigen expression between primary and metastatic tumours was noticed in a few cases (one colonic adenocarcinoma in frozen sections with OV-TL3 and four ovarian adenocarcinomas in paraffin wax sections with OC125, parlam-4, and 115 D8).

\section{FROZEN MATERIAL}

The antibodies giving a significant result $(p<0.05)$ in the univariate discrimination between colonic and ovarian adenocarcinomas in frozen sections of the training set are listed in table 2. OV-TL3, OC125, and parlam-4 were the most significant discriminating antibodies in both statistical tests $(p<0.0002)$.

For selection of a combination of antibodies, stepwise linear discriminant analysis was carried out, using equal a priori probabilities for ovarian and colonic adenocarcinomas. The stepwise selection of antibodies is based on the higher $F$ ratio in the discriminant analysis.

The best discriminative antibody for frozen sections in the training set was OV-TL3. The results using OC125 showed that it had an additional discriminative contribution. For clinical management high posterior probabilities are mandatory. The ovarian posterior probability is the probability of classifying a carcinoma in the ovarian group. The sum of the ovarian posterior probability and the colonic posterior probability is always 1.0 , and therefore, the values 0.1 and 0.9 were selected as cut off points for the posterior probabilities (jackknifed classification method). With
Table 2 Univariate analysis of staining results with monoclonal antibodies applied on frozen sections from ovarian and colonic adenocarcinomas in training set

\begin{tabular}{lll}
\hline Antibody & $\begin{array}{c}p \text { Value (Wilcoxon's } \\
\text { non-parametric test) }\end{array}$ & $p$ Value $\left(\chi^{2}\right.$ test) \\
\hline OC125 & 0.0000 & 0.0000 \\
OV-TL3 & 0.0000 & 0.0000 \\
19.9 & 0.2136 & 0.8949 \\
Parlam-4 & 0.0002 & 0.0000 \\
Anti-secretory & & \\
component & 0.0329 & 0.0316 \\
115 D8 & 0.0000 & 0.0635 \\
67 D11 & 0.3275 & 0.0359 \\
125 B5 & 0.0052 & 0.0001 \\
B 72.3 & 0.9357 & 0.3558 \\
\hline
\end{tabular}

Significance $=p<0.05$.

the jackknifed classification method each carcinoma was classified by criteria based on all other carcinomas of the group. This procedure was repeated for each carcinoma in the group until all carcinomas were classified. Fig 1(a) shows the ovarian posterior probabilities for ovarian adenocarcinomas and colonic adenocarcinomas. Both ovarian and colonic adenocarcinomas were recorded as "non-classifiable" when the posterior probability lay between $0 \cdot 1$ and 0.9 . An adenocarcinoma is predicted as having its origin in the colon when the ovarian posterior probability is less than $0 \cdot 1$ and as ovarian adenocarcinoma when the ovarian posterior probability is greater than 0.9 . Fig la shows that 16 of $24(67 \%)$ of the ovarian adenocarcinomas and 16 of $20(80 \%)$ of the colonic adenocarcinomas were correctly classified. Of the eight ovarian adenocarcinomas incorrectly classified, six were of the mucinous type. Two of these six mucinous tumours had been incorrectly classified as of colonic origin. Three of the eight incorrectly classified ovarian adenocarcinomas were predicted to be of ovarian origin with posterior probabilities of $>0.7$. More interesting are the results of predicting a colonic origin of an adenocarcinoma. Not one metastatic colonic adenocarcinoma was predicted to be of ovarian origin, with an ovarian posterior probability of $>0.9$.

Antibodies selected in the training set by their discriminatory power in stepwise linear discriminant analysis were subsequently applied on the test set. Jackknifed classification results of the specimens with the selected antibodies are shown in fig 1(b), which gives the ovarian posterior probabilities for ovarian adenocarcinomas and colonic adenocarcinomas. Ten of $17(59 \%)$ of the primary ovarian adenocarcinomas and 17 of $21(81 \%)$ of the primary colonic adenocarcinomas were correctly classified.

Seven ovarian adenocarcinomas were not correctly classified. Five of the seven were non-classifiable, but three of these five were predicted to be of ovarian 

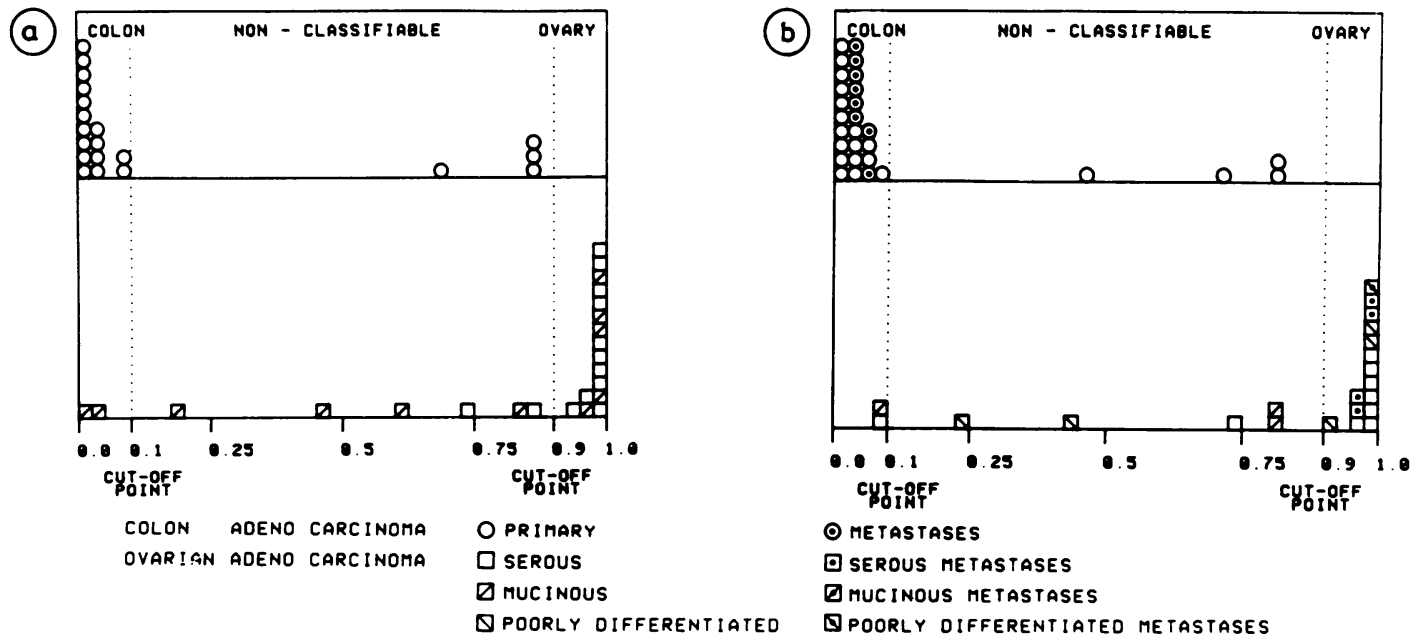

Fig 1 Computer classification according to staining results of $O V-T L 3$ and $O C 125$ on frozen sections of training set (a) and test set (b) using ovarian posterior probabilities of $0 \cdot 1$ and 0.9 as cut off points.

origin with ovarian posterior probabilities of $>0.7$. Two ovarian adenocarcinomas were predicted to be of colonic origin. Of the seven incorrectly classified ovarian adenocarcinomas, two were serous tumours, three were mucinous tumours, one a mixed serous/ mucinous tumour and one an endometrioid tumour. Three of the four "non-classifiable" colonic adenocarcinomas had ovarian posterior probabilities of $>$ $0 \cdot 7$. They were histologically moderately $(n=1)$ or poorly differentiated $(n=2)$. What is remarkable is that all metastatic ovarian $(n=5)$ and colonic $(n=8)$ adenocarcinomas were correctly classified.

PARAFFIN WAX EMBEDDED MATERIAL

The best discriminatory antibodies in paraffin wax sections selected by univariate statistical analysis in the training set are shown in table 3. Parlam-4 and OC125 gave the most significant results in both

Table 3 Univariate analysis of staining results with monoclonal antibodies applied on paraffin sections from ovarian and colonic adenocarcinomas in training set

\begin{tabular}{lll}
\hline Antibody & $\begin{array}{l}\text { p Value (Wilcoxon's } \\
\text { non-parametric test) }\end{array}$ & $p$ Value ( $\chi^{2}$ test) \\
\hline OC125 & 0.0013 & 0.0010 \\
19.9 & 0.4185 & $*$ \\
Parlam-4 & 0.0000 & 0.0000 \\
Anti-secretory & & \\
component & 0.0015 & 0.0028 \\
115 D8 & 0.0000 & 0.8481 \\
67 D11 & 0.0399 & 0.2678 \\
B 72.3 & 0.0233 & $*$ \\
\hline
\end{tabular}

Significance $=p<0.05$.

* No statistics computed; the contingency table had less than two rows or two columns. statistical tests. $115 \mathrm{D} 8$ also gave significant results ( $p<0.0001$ ) by Wilcoxon's non-parametric test.

Stepwise linear discriminant analysis, assuming equal a priori probabilities for ovarian and colonic adenocarcinomas, indicated that parlam -4 was the most discriminatory antibody from the training set. OC125 and 115 D8, although both significantly discriminative antibodies in the univariate analysis, gave less significant results in the multivariate analysis than parlam-4. Fig 2(a) gives the results of the jackknifed classification on paraffin wax embedded material of colonic and ovarian adenocarcinomas. As in frozen material ovarian posterior probabilities of 0.9 and 0.1 were used as cut off points to place patients correctly in the ovarian adenocarcinoma or colonic adenocarcinoma groups.

Both ovarian and colonic adenocarcinomas were recorded as "non-classifiable" when the posterior probability lay between $0 \cdot 1$ and $0 \cdot 9$. Fig $2 a$ shows that 17 of $24(71 \%)$ of the ovarian adenocarcinomas and 18 of $20(90 \%)$ of the colonic adenocarcinomas were correctly classified. Five ovarian adenocarcinomas, all of the mucinous type, were incorrectly classified as belonging to the colonic group; one colonic adenocarcinoma was classified as belonging to the ovarian group. Two ovarian adenocarcinomas were recorded as "non-classifiable" with an ovarian posterior probability of $>\mathbf{0 . 7}$; one colonic adenocarcinoma was recorded as "non-classifiable" with an ovarian posterior probability of $<0.25$.

The efficacy of antibody parlam-4, selected by stepwise linear discriminant analysis, was applied to the test set. Jackknifed classification results are shown in fig 2(b). Sixteen of $23(70 \%)$ of the primary ovarian 

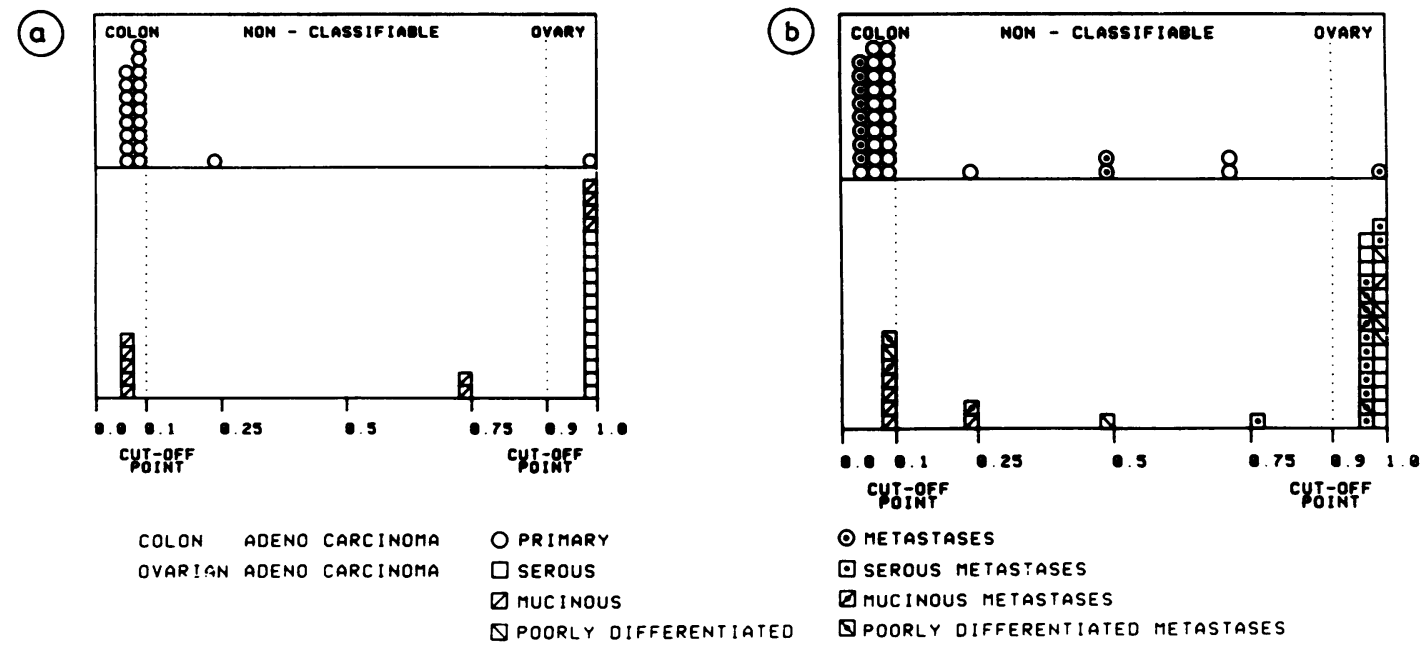

Fig 2 Computer classification according to staining results of parlam-4 on paraffin wax sections (a) test set and training set (b) using ovarian posterior probabilities of 0.1 and 0.9 as cut off points.

adenocarcinomas and 21 of $24(88 \%)$ of the primary colonic adenocarcinomas were correctly classified. Thirteen of $17(76 \%)$ of the metastatic ovarian tumours and eight of $11(73 \%)$ of the metastatic colonic adenocarcinomas were correctly classified. Of the four ovarian tumours recorded as "non-classifiable" and the seven incorrectly classified as colonic, seven were mucinous adenocarcinomas, one a serous adenocarcinoma, and three were indeterminate adenocarcinomas. Of the seven incorrectly classified ovarian adenocarcinomas, five were primary lesions and two metastases.

Of the five colonic adenocarcinomas recorded as non-classifiable, three were primary lesions and two metastases. Only one (metastatic) colonic adenocarcinoma was incorrectly classified.

\section{Discussion}

This study shows that none of the antibodies by itself was specific enough to be able to predict either colonic or ovarian origin of metastatic adenocarcinoma. The best discrimination between colonic and ovarian adenocarcinomas was obtained by using combinations of antibodies selected by linear stepwise discriminant analysis.

For frozen material OV-TL3 and OC125 were best. This is in line with previous reports in which no reactivity of colonic adenocarcinomas with OV-TL3 ${ }^{5}$ and only incidentally with OC125 was found..$^{2-4}$ For paraffin wax embedded material parlam-4 was the best. Although $\mathrm{OC125}$ and $115 \mathrm{D} 8$ individually had discriminative power in univariate analysis, they did not contribute significantly to the classification obtained by parlam- 4 .

In general, antigens present in relatively high concentrations within the cytoplasm are well preserved during fixation and embedding procedures. By contrast, surface antigens are often denatured or masked, and are better preserved in frozen sections, so antibodies reactive to membrane bound antigens such as monoclonals $115 \mathrm{D} 8,67 \mathrm{D} 11,125 \mathrm{~B} 5, \mathrm{~B} 72.3,19.9$, parlam-4, OV-TL3 and OC125 all show varying degrees of loss of antigenic reactivity. For example, the reactivity of OV-TL3 and 125 B5 is completely destroyed; that of OC125 only partially; and the reactivity of parlam-4, $115 \mathrm{D} 8,67 \mathrm{D} 11, \mathrm{~B} 72.3$ and 19.9 is still well enough preserved to obtain reliable staining results. These differences in loss of antigenic reactivity might explain why different combinations of antibodies are suitable for frozen and paraffin wax embedded material. Caution is needed in interpreting staining results obtained on paraffin wax embedded sections with antibodies that have been selected for their specificity on frozen sections.

Considering the results of the computer classification of ovarian and colonic adenocarcinomas with OV-TL3 and OC125 on frozen material, no reliable results were obtained for the classification of ovarian adenocarcinomas - that is, there were too many tumours incorrectly predicted to be of colonic origin (ovarian posterior probability of $<0 \cdot 1$ ). By contrast, no colonic adenocarcinoma was incorrectly predicted to be of ovarian origin (ovarian posterior probability of $>0.9$ ). Thus if the method is used for differentiating between colonic or ovarian origin, the computer classification as an ovarian adenocarcinoma (ovarian posterior probability of $>0.9$ ) excludes the possibility 
of a colonic adenocarcinoma. This is important as ovarian adenocarcinomas are treated with high doses of chemotherapy including cisplatin: computer classification prevents overtreatment of the patient.

The same trend is apparent when considering the paraffin wax embedded material. In the group of ovarian adenocarcinomas: 10 of $47(21 \%)$ primary tumours and two of $17(12 \%)$ metastatic tumours were incorrectly predicted to be of colonic origin. In the group of colonic adenocarcinomas only one of 44 $(2 \%)$ primary tumours and one of $11(9 \%)$ metastatic tumours were incorrectly predicted to be of ovarian origin. In both groups (ovarian and colonic) four of 47 $(9 \%)$ primary ovarian tumours, two of $17(12 \%)$ ovarian metastases, four of $44(9 \%)$ primary colonic tumours and two of $11(18 \%)$ metastases of colonic tumours were recorded as "non-classifiable".

Mucinous ovarian adenocarcinomas most often caused diagnostic problems in the computer classification: 12 of 14 primary and one of two metastatic tumours incorrectly predicted to be of colonic origin turned out to be mucinous adenocarcinomas.

As mucinous ovarian carcinomas pose the most problems in determining the organ of origin we also analysed the antigen profiles of the mucinous ovarian carcinomas and colonic carcinomas to see whether the classification results could be improved for mucinous ovarian carcinomas. The same antibodies were selected by the stepwise discriminate analysis as for the whole group of ovarian carcinomas for frozen and paraffin wax embedded material. Moreover, the classification results for mucinous carcinomas were similar to those based on the statistical analysis of the whole group of ovarian carcinomas (data not shown).

In conclusion, this preliminary study shows that the statistical analysis of antigen profiles in frozen as well as in paraffin wax embedded tissue sections can be helpful in differentiating colonic from ovarian adenocarcinomas.

We thank Thea Tadema, Wim Vos, and Gijsbert Heuff for their technical assistance, Hans Vuik for the graphical work, and Sylvia Haverkamp and Aloysia Sugiarsi for preparing the manuscript.

\section{References}

1 Serov SF, Scully RE. Histological typing of ovarian tumours. International histological classification of tumours. No 9. Geneva: World Health Organisation, 1973.

2 Bast RC, Feeney M, Lazarus H, et al. Reactivity of a monoclonal antibody with human ovarian carcinomas. $J$ Clin Invest 1981;68:1331-7.
3 Kabawat SE, Bast RC, Welch WR, et al. Immunopathologic characterization of a monoclonal antibody that recognizes a common surface antigen of human ovarian tumors of serous, endometrioid and clear cell type. Am J Clin Pathol 1983;79: 98-104.

4 Koelma IA, Nap M, Rodenburg CJ, Fleuren GJ. The value of tumour marker CA125 in surgical pathology. Histopathology 1987;11:287-94.

5 Poels LG, Peters D, van Megen Y, et al. Monoclonal antibody against human ovarian tumor-associated antigens. JNCI 1986;75:781-91.

6 Herlyn M, Steplewski Z, Herlyn D, et al. Colorectal carcinomaspecific antigen: detection by means of monoclonal antibodies. Proc Natl Acad Sci USA 1979;76:1438-42.

7 Verstijnen CPHJ, Arends JW, Moerkerk PTM, et al. CEAspecificity of CEA-reactive monoclonal antibodies. Immunochemical and immunocytochemical studies. Anticancer Res 1986;6:97-104.

8 Harris JP, South MA. Secretory component, a glandular epithelial cell marker. Am J Pathol 1981;105:47-53.

9 Hilkens J, Buys F, Hilgers J, et al. Monoclonal antibodies against human milk fat globule membranes detecting differentiation antigens of the mammary gland and its tumors. Int $J$ Cancer 1984;34:197-206.

10 Hilkens J, Kroezen V, Buys F, et al. MAM-6, a carcinoma associated marker; preliminary characterization and detection in sera of breast cancer patients. In: Ceriani RL, ed. Monoclonal antibodies and breast cancer. Boston: Martinus Nijhoff, 1984:28-42.

11 Gooi HC, Jones NJ, Hounsell EF, et al. Novel antigenic specificity involving the blood group antigen $\mathrm{LE}^{\mathrm{a}}$, in combination with oncodevelopmental antigen SSEA-1 recognized by two monoclonal antibodies to human milk fat globule membranes. Biochem Biophys Res Comm 1985;131:543-50.

12 Gooi HC, Jones NJ, Hilkens J, et al. Lewis blood group-related specificities of monoclonal antibodies designated MAM 3a, b and c against human milk fat globule membranes. Glycoconjugate 1985;2:409-17.

13 Gerö E, Hageman PH, Hilkens J, et al. Diversity of antigenic expression on normal and malignant human mammary cells recognized by a new panel of monoclonal antibodies. In: Ceriani R, ed. Immunological approaches to the diagnosis and therapy of breast cancer. Proceedings of Second International Workshop on Monoclonal Antibodies and Breast Cancer, San Francisco, November 20-21, 1986. New York: Plenum.

14 Colcher D. A spectrum of monoclonal antibodies reactive with human mammary tumor cells. Proc Natl Acad Sci USA 1981;78:3199-203.

15 Nuti M. A monoclonal antibody (B 72.3) defines patterns of distribution of a novel tumor associated antigen in human mammary carcinoma cell populations. Int J Cancer 1982; 29:539-45.

16 Logmans SC, Jöbsis AC. Thyroid associated antigens in routinely embedded carcinomas. Possibilities and limitations studied in 116 cases. Cancer 1984;54:274-9.

17 Henzen-Logmans SC, Mullink H, Ramaekers FCS, et al. Expression of cytokeratins and vimentin in epithelial cells of normal and pathologic thyroid tissues. Virchows Arch (Pathol Anat) 1987; $410: 347-54$.

Requests for reprints to: Dr S C Henzen-Logmans, Department of Pathology, Free University, De Boelelaan 1117, 1081 HV Amsterdam, The Netherlands. 